\title{
DETERMINACIÓN DE LA EFICIENCIA DEL PROCESO DE FERMENTACIÓN REALIZADO POR LAS LEVADURAS Candida kefyry Saccharomyces cerevisiae EN LA PRODUCCIÓN DE XILITOL
}

\author{
DETERMINATION OF THE EFFICIENCY OF THE FERMENTATION PROCESS BY \\ YEAST CANDIDA KEFYR AND SACCHAROMYCES CEREVISIAE IN THE \\ PRODUCTION OF XILITOL.
}

Henry Reyes Pineda ${ }^{1}$, Bibiana Rosero ${ }^{2}$, Leidy Carolina Cardona ${ }^{3}$.

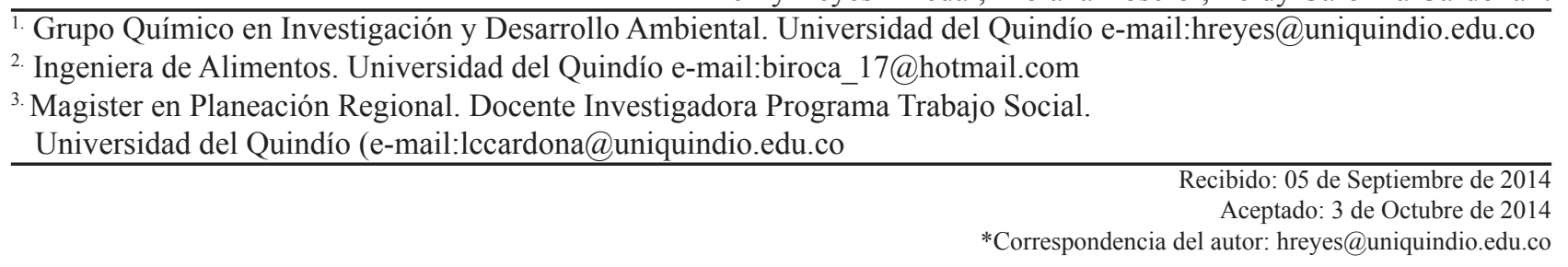

\section{RESUMEN}

Este trabajo consistió en determinar la eficiencia fermentativa de las levaduras Candida kefyr y Saccharomyces cerevisiae en hidrolizado de hojas de Eucalyptus camaldulensis Dehnh. (Myrtaceae) para la producción de xilitol. Para la caracterización fisicoquímica del hidrolizado del material vegetal se midieron: humedad, acidez, grados brix, $\mathrm{pH}$, densidad, azúcares reductores, análisis termogravimétrico (TGA), infrarrojo. Los análisis: de humedad, $\mathrm{Aw}, \mathrm{y}$ de grados brix, arrojaron valores de: 4.29, 0,45 y 4,03, respectivamente. El análisis termogravimétrico mostró que hasta los $200^{\circ} \mathrm{C}$ E. camaldulensis es relativamente estable y que a temperaturas superiores los principios activos empiezan a degradarse. La hidrólisis del material vegetal se realizó por ultrasonido a $37^{\circ} \mathrm{C}$ por 20 minutos. El proceso fermentativo se realizó a una temperatura de $35+/-2{ }^{\circ} \mathrm{C}, 200 \mathrm{rpm}$ por un período de 30 horas, utilizándose las levaduras Candida kefyr y Saccharomyces cerevisiae, y realizando análisis de azúcares reductores por DNS. Para ambas levaduras el resultado del análisis de azúcares reductores mostró que entre mayor tiempo de fermentación menor concentración de xilosa en el hidrolizado, resultado que indica que las levaduras utilizaron xilosa para producir xilitol. Saccharomyces Cerevisiae presentó mejor eficiencia consumiendo 51,42 \% de la xilosa, en contraste, Cándida kefyr consumió $36,81 \%$, ambas en un período de 30 horas.

Palabras claves: Xilosa, Xilitol, fermentación, Eucalyptus camaldulensis, Saccharomyces Cerevisiae, Candida kefyr.

\section{ABSTRACT}

This study consisted of determining the efficiency of fermentative yeasts Saccharomyces Cerevisiae and Candida Kefyr hydrolyzate leaves of Eucalyptus Camaldulensis Dehnh. (Myrtaceae) for xylitol production. For the physicochemical characterization of hydrolyzed plant, material humidity, acidity, degrees brix, $\mathrm{pH}$, density, reducing sugars, thermogravimetric analysis (TGA), and infrared were measured.. Analysis: the results of moisture, Aw, and brix degrees were: $4.29,0.45$ and 4.03, respectively. Thermogravimetric analysis showed that E. Camaldulensis is relatively stable at higher temperatures (up to $200^{\circ} \mathrm{C}$ ) and the active ingredients start to degrade. The hydrolysis of plant material was carried out by ultrasound at $37^{\circ} \mathrm{C}$ for 20 minutes. The fermentation process was performed at a temperature of $35+/-2^{\circ} \mathrm{C}, 200 \mathrm{rpm}$ for a 30 hour period, using the yeast Saccharomyces Cerevisiae and Candida Kefyr, and performing analysis of reducing sugars by the DNS. For both yeast analyses, the results showed that reducing sugars between lower longer fermentation xylose concentrations in the hydrolysate. This result indicates that the yeast was used xylose to produce xylitol. Saccharomyces Cerevisiae showed better efficiency consuming xylose $51.42 \%$. Incontrast, Candida Kefyr consumed $36.81 \%$, both over a period of 30 hours.

Keywords: Xilosa, Xilitol, sweetener, hydrolysis, Eucalyptus camaldulensis, Saccharomyces Cerevisiae, Candida kefyr. 


\section{INTRODUCCIÓN}

Colombia nos está llevando a tendencias de salud y bienestar evidenciándose en lo que se conoce hoy en día como alimentos funcionales, que brindan seguridad y bienestar a los consumidores. Hay un amplio campo de estudio sobre las diferentes especies o variedades del género puesto que en Colombia solo es utilizado un porcentaje muy reducido de esta planta; estudios realizados han demostrado las bondades que esta especie ofrece a la salud y la alimentación. El estudio realizado se basó en la obtención de xilitol a partir del metabolismo de la xilosa, uno de los azúcares presentes en las hojas de Eucalyptus camaldulensis Dehnh. (Myrtaceae) evaluando la eficiencia de dos tipos de levaduras durante el proceso de fermentación o la transformación de xilosa a xilitol.

Los representantes de Eucalyptus camaldulensis Dehnh. (Myrtaceae) son Arboles, 5-12 (-45) m de alto, corteza lisa, blanca, gris, café o roja; ramas jóvenes glabras. Hojas juveniles no prevalecientes, ovadas a anchamente lanceoladas; hojas adultas alternas, angostamente lanceoladas, 9-22 cm de largo y 1-2.5 cm de ancho, comúnmente falcadas, ápice agudo, base cuneada, glabras, pecíolo no torcido. Inflorescencias umbelas axilares, 6-11 flores por umbela, yemas lanceoladas u ovoide-apiculadas, ca $5 \mathrm{~mm}$ de largo; cáliz operculado. Cápsula cupuliforme, 5 (-8) mm de largo, disco ascendente, valvas (3) 4 (5), exertas (tomado de http://www.tropicos.org diciembre 12 de 2012). Esta especie es ampliamente utilizada como ornamental siendo ampliamente cultivada en los trópicos y subtrópicos.

Para este estudio el material vegetal utilizado correspondió a láminas foliares de E. camaldulensis. Dado que la mayor presencia de metabolitos en las hojas está presente en la madrugada, las muestras fueron recolectadas en este momento del día. Posteriormente fueron sometidas a un proceso de deshidratación intervalos de tiempo, hasta obtener material seco, es importante mencionar que la humedad puede causar deterioro del material vegetal que será utilizado en la fase experimental. Las muestras procesadas fueron sometidas a un proceso fisicoquímico con el fin de caracterizar e identificar con precisión la especie vegetal. Luego fueron llevadas a un proceso de hidrólisis ácida por medio de ultrasonido a $37^{\circ} \mathrm{C}$, para después ser detoxificado, clarificado y neutralizado, ajustando su $\mathrm{pH}$ entre 4-4,5 con el fin de que fuera apropiado para la adecuación de las levaduras.

En la presente investigación se seleccionaron dos tipos de levaduras, Saccharomyces Cerevisiae y Candida Kefyr las cuales se comportaron bien en investigaciones relacionadas con procesos de fermentación. Estas levaduras se incubaron durante 24 horas en un agua de peptona; posteriormente se inocularon en el hidrolizado previamente esterilizado en una relación $70: 30 \mathrm{v} / \mathrm{v}$ para luego ser sometidas a procesos de fermentación.

El objetivo de este trabajo de investigación consistió en determinar la eficiencia de cada una de las levaduras en la producción de xilitol, caracterizando fisicoquímicamente cada una de sus etapas de producción.

\section{MATERIALES Y MÉTODOS}

El estudio se realizó en Armenia, Quindío en los laboratorios de la Universidad del Quindío (laboratorio análisis instrumental, Bloque ciencias básicas; invitro, plantas piloto, laboratorio de fitoquímica), así mismo se realizaron algunas pruebas en el laboratorio de Cromatografía de la Universidad de Caldas.

\subsection{Fases 0 etapas}

\subsubsection{Recolección del material vegetal}

El material vegetal fue recolectado en la finca La Chena vía a Cruces, municipio de Filandia en horas de la mañana. Para la toma de las muestras se contó con el permiso de la Universidad del Quindío y gestionado por el Centro de Investigaciones en BiodiversidadCIBUQ.

\subsubsection{Tratamiento del material vegetal}

Se adecuaron muestras de 2.0 por $4.0 \mathrm{~cm}$, las cuales fueron secadas en un horno de recirculación a $37{ }^{\circ} \mathrm{C}$ en intervalos de tiempo correspondientes a 24,48 y 72 horas hasta obtener material seco y deshidratado.

\subsubsection{Caracterización fisicoquímica del material vegetal}

Para los análisis fisicoquímicos de la planta se procedió a moler finamente los fragmentos de las hojas, realizándose los siguientes análisis: Humedad, Actividad de agua, Grados brix, TGA y FTIR.

\subsubsection{Caracterización microbiológica de $E$. camal- dulensis}

Análisis de mohos y levaduras, Se realizó en agar Ogy (Agar-Oxitetraciclina-glucosa-extracto de leva- 
dura) sembrando diluciones 10-1, 10-2 y 10-3 por duplicado a profundidad. Mesófilos, se realizó en Plate Count agar $(P C A)$ realizando diluciones 10-1, 10-2 y 10-3 por duplicado y a profundidad. Coliformes totales y fecales, se realizó por la técnica del número más probable (NMP) utilizando Caldo Brila, triptófano y reactivo de Covac's.

\subsubsection{Hidrólisis del sustrato en base ácida}

La hidrólisis ácida se llevó a cabo utilizando catalizadores ácidos para transformar las cadenas de polisacáridos que conforman las hojas de eucalipto (hemicelulosa y celulosa en sus monómeros elementales) para ello se utilizó dos metodologías una por alta presión (autoclave) y otra por medio de ultrasonido. Las proporciones de ácido y material vegetal utilizadas fueron las descritas por Villalobos Cortinez, (2010), tomando una solución de ácido sulfúrico a una concentración del $1 \%$, en una relación de 1:10 (peso) de material vegetal a tratar y ácido sulfúrico.

- La primera hidrólisis ácida se llevó a cabo mediante utilización de la técnica descrita por Villalobos Cortinez (2010), en el cual se llevó el material vegetal en proporción de 1:10 a un autoclave, durante un tiempo de 30 minutos a una temperatura de $120^{\circ} \mathrm{C}$ y $15 \mathrm{PSI}$; las muestras fueron extraídas y enfriadas en baño de hielo para detener la reacción. Después de esto se realizó un filtrado y centrifugado del hidrolizado por 10 minutos a $150 \mathrm{rpm}$, para una posterior caracterización fisicoquímica.

- La segunda hidrólisis ácida se realizó por $20 \mathrm{mi}-$ nutos a una temperatura de $37^{\circ} \mathrm{C}$, por ultrasonido. El hidrolizado obtenido se filtró y centrifugó por 10 minutos a $150 \mathrm{rpm}$ y se le realizó la caracterización fisicoquímica correspondiente.

Los hidrolizados fueron caracterizados fisicoquímicamente mediante: Cuantificación de azúcares reductores, (método de DNS), pH, Acidez, Grados brix, Densidad, FTIR y Calor latente.

\subsubsection{Detoxificación del hidrolizado}

El hidrolizado obtenido se concentró en un Rotavapor a $70 \pm 4{ }^{\circ} \mathrm{C}$ hasta retirar un $60 \%$ de agua del hidrolizado. Se realizó una sobretitulación del hidrolizado en donde se aumentó el $\mathrm{pH}$ a 10, con hidróxido de sodio $(\mathrm{NaOH})$ sólido, posteriormente se disminuyó el $\mathrm{pH}$ a 5,8, empleando ácido sulfúrico (H2SO4). Durante la sobretitulación con hidróxido de sodio y ácido sulfú- rico se realizó filtración del precipitado formado en el hidrolizado. Otro procedimiento para detoxificar el hidrolizado rico en xilosa fue el tratamiento con carbón activado, el cual consistió en someter la solución a una clarificación con $2,5 \%$ de carbón activado a 200 rpm y $30{ }^{\circ} \mathrm{C}$ durante una hora. Luego se filtró y centrifugó. (Herazo Camaño, Cárdenas Ruiz, \& Arrazola Paternina, 2011).

\subsubsection{Proceso Fermentativo}

2.1.7.1. Preparación de cepas. Medios de cultivo y confirmación de crecimiento de colonias. Para el aislamiento y determinación de la cantidad de levadura adecuada a inocular en el hidrolizado se utilizó el medio de cultivo Sabouraud en agar y agua de peptona líquido esterilizado, al cual se adicionó gentamicina al $10 \%$, con el fin de evitar el crecimiento bacteriano. (Vanegas et al. 2004) Se realizó una dilución de 0,16 gr de levadura Saccharomyces cerevisiae en $30 \mathrm{~mL}$ de agua de peptona y $0.16 \mathrm{~mL}$ de levadura Candida kefyr en $30 \mathrm{~mL}$ de agua de peptona, se realizó una siembra por estría para confirmar el crecimiento y viabilidad de las cepas, de $S$. cerevisiae y $C$. kefyr. Se incubaron en la oscuridad por un período de 7 días a temperatura ambiente.

\subsubsection{Preparación del inóculo}

Se inocularon 0,16 gramos de $S$. cerevisiae levadura activa seca y $0,16 \mathrm{~mL}$ de $C$. kefyr (granos kefyr) en $30 \mathrm{~mL}$ de agua de peptona y se incubó a $35^{\circ} \mathrm{C}$ por 24 horas respectivamente, para ser inoculados en el sustrato.

\subsubsection{Esterilización de sustrato}

El sustrato se esterilizó en autoclave a una temperatura de $120^{\circ} \mathrm{C}$ y una presión de 15 PSI durante 20 minutos, este procedimiento se realizó con el objetivo de controlar el crecimiento microbiano (virus y esporas) que pudieran competir con las cepas a inocular en el sustrato.

\subsubsection{Inoculación}

Para la inoculación de la levadura en el hidrolizado se realizó una mezcla en relación $70: 30 \mathrm{v} / \mathrm{v}$ de hidrolizado de eucalipto detoxificado con un ajuste de $\mathrm{pH}$ de 4,5 previamente esterilizado, y agua de peptona con levadura inoculada previamente incubada; después de la mezcla se confirmó el pH y se llevó a fermentación. La inoculación se realizó en cabina de flujo laminar. 


\subsubsection{Fermentación}

La fermentación se realizó bajo las siguientes condiciones: temperatura de $35+/-2{ }^{\circ} \mathrm{C}$ (González-Hernández, 2011) a $200 \mathrm{rpm}$ por 30 horas (Vanegas et al. 2004) durante las cuales se tomaron muestras del fermentado en intervalos de 0, 6, 12, 24 y 30 horas de fermentación. El proceso se realizó en un fermentador artesanal que fue diseñado y ubicado en el laboratorio de microbiología del edificio de Plantas piloto de la Universidad del Quindío.

\subsubsection{Análisis de la producción de xilitol}

Para verificar el comportamiento de la xilosa durante el proceso de fermentación se realizaron análisis de DNS, en períodos de 6, 12, 24 y 30 horas de fermentado. Para confirmar la producción de xilitol durante el proceso fermentativo, se utilizó la técnica de HPLC para lo cual se extrajeron muestras en diferentes tiempos $(0,6,24$, y 30 horas $)$ a las cuales se les realizó un procedimiento de dilución y eliminación de proteínas, lípidos y demás compuestos de alto peso molecular, posteriormente fueron microfiltradas a través de membranas de $0.45 \mu \mathrm{m}$, luego fueron trasladadas hacia el laboratorio de análisis instrumental de la Universidad de Caldas con el fin de realizar el análisis de la producción de xilitol mediante la técnica de HPLC para lo cual se utilizó un detector de índice de refracción (IR) y la columna varian carbohydrates $\mathrm{Ca}$, en las siguientes condiciones: efluente $\mathrm{H}_{2} \mathrm{O}$ flujo de 0.5 $\mathrm{mL} / \mathrm{min}$, temperatura de la columna $90{ }^{\circ} \mathrm{C}$, atenuación en el detector 8 , volumen de la muestra $20 \mu \mathrm{L}$ (Vanegas et al. 2004).

Para comparar cuál de las levaduras (C. kefyr y S. cerevisiae) presenta mayor eficiencia en el proceso de fermentación para la producción de xilitol, se utilizó un análisis estadístico de regresión lineal simple.

La figura 1 muestra el termograma de la muestra vegetal; la curva de color azul representa el cambio porcentual de masa de E. Camaldulensis con relación al aumento de la temperatura. La curva verde corresponde a la primera derivada que permite evidenciar el punto real de degradación de la muestra, es decir, el cambio de la masa con relación al tiempo o la temperatura; la presencia de picos permite determinar el número de etapas de descomposición de la muestra. El termograma evidencia 3 picos de descomposición de la muestra, la principal pérdida se presenta a una temperatura de $200{ }^{\circ} \mathrm{C}$ o superior a esta con un porcentaje del $25,35 \%$ en adelante, este resultado indica que la

\section{RESULTADOS Y DISCUSIÓN}

3.1. Caracterizacion fisicoquimica del material vegetal 3.1.1. Análisis termogravimétrico TGA

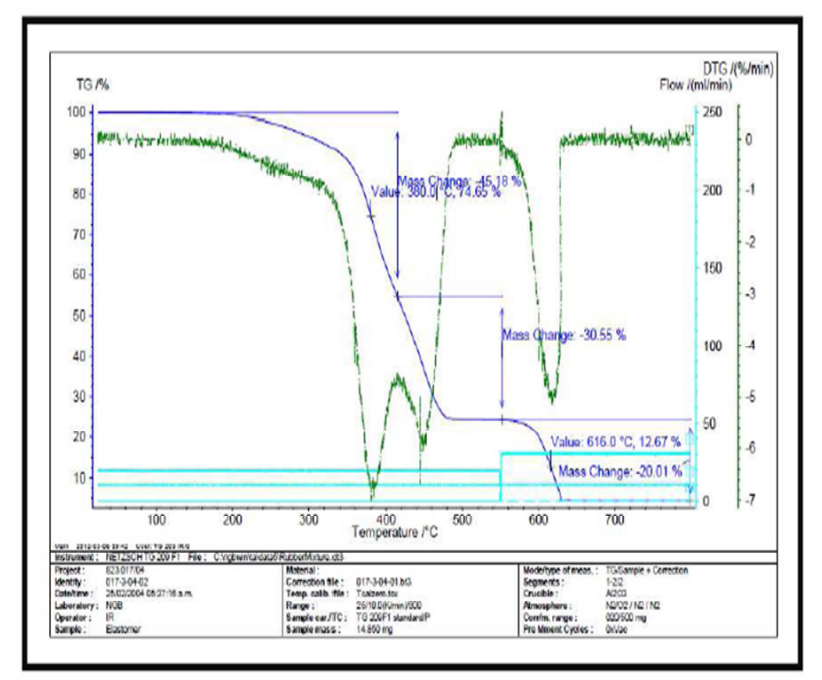

Figura 1: Análisi termigravimetrico (TGA) de $E$. Camaldulensis.

estabilidad se pierde cuando excede esta temperatura. La figura 1 muestra el termograma de la muestra vegetal; la curva de color azul representa el cambio porcentual de masa de E. Camaldulensis con relación al aumento de la temperatura. La curva verde corresponde a la primera derivada que permite evidenciar el punto real de degradación de la muestra, es decir, el cambio de la masa con relación al tiempo o la temperatura; la presencia de picos permite determinar el número de etapas de descomposición de la muestra. El termograma evidencia 3 picos de descomposición de la muestra, la principal pérdida se presenta a una temperatura de $200{ }^{\circ} \mathrm{C}$ o superior a esta con un porcentaje del $25,35 \%$ en adelante, este resultado indica que la estabilidad se pierde cuando excede esta temperatura.

\subsubsection{Infrarrojo por Transformada de Fourier, FTIR de la muestra vegetal.}

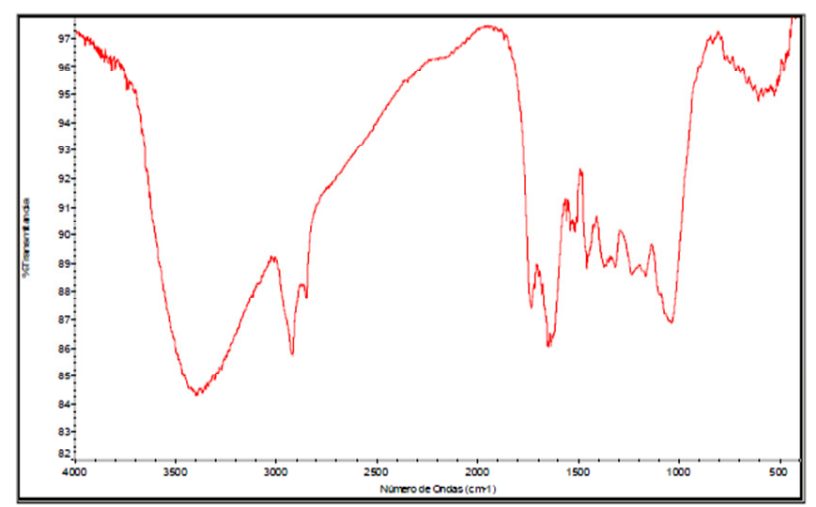

Figura 2: Espectro infrarrojo Eucalyptus camaldulensis 
La figura 2 muestra el espectro infrarrojo tomados en $\mathrm{KBr}$ del E. Camaldulensis en el cual se obtiene la información estructural del material, en este se observa una banda con frecuencia de vibración de $3400 \mathrm{~cm}^{-1}$, correspondiente a los grupos- $\mathrm{OH}$ (diferente al grupo $\mathrm{OH}$ del agua) el cual es el representativo e indica la presencia de alcoholes en este tipo de material vegetal.

\subsubsection{Características fisicoquímicas del E. Camal- dulensis}

Tabla 1: Resultados del análisis fisicoquímico de características $E$. camaldulensis

\begin{tabular}{cc}
\hline Característica & Valor \\
\hline Humedad inicial & $53.4 \%$ \\
Humedad Final & $4.29 \%$ \\
Aw & 0.4548 \\
${ }^{\circ}$ Brix & 4 \\
TGA & Figura 1 \\
FTIR & Figura 2 \\
\hline
\end{tabular}

\subsubsection{Caracterización fisicoquímica de hidroliza-} dos

\subsubsection{Hidrólisis ácida (autoclave)}

En la Tabla 2 se muestran los diferentes valores de los análisis fisicoquímicos realizados a la hidrólisis obtenida por medio de autoclave.

Tabla 2: Propiedades fisicoquímicas del hidrolizado de Eucaliptus camaldulensis obtenido por hidrólisis ácida en autoclave

\begin{tabular}{cc}
\hline HIDRÓLISIS ÁCIDA EN AUTOCLAVE \\
\hline o Brix & 5.02 \\
$\mathrm{pH}$ & 2.31 \\
$\%$ Acidez & 1.86 \\
Densidad & $1.27 \mathrm{~g} / \mathrm{mL}$ \\
FTIR (Bagazo) & Figura 3 \\
\hline
\end{tabular}

El hidrolizado obtenido por autoclave arrojó un resultado de grados brix de 5.02, lo cual evidencia que en el proceso de hidrolizado se presentó un aumento significativo con respecto al material seco. En cuanto al $\mathrm{pH}$ el valor obtenido fue de 2.31 el cual tiene relación con el solvente en el cual se hizo la hidrólisis (ácido sulfúrico $1 \%$ ), el valor de acidez del hidrolizado arrojó un resultado de $1,86 \%$ teniendo en cuenta que para este caso el ácido predominante es el áci- do sulfúrico; en cuanto a la densidad el resultado fue de 1,271 gr/mL, valor necesario para el posterior cálculo del calor latente del proceso.

\subsubsection{Análisis Infrarrojo Autoclave del hi- drolizado}

En la figura 3 se evidencia el comportamiento del material vegetal después del procedimiento de hidrólisis.

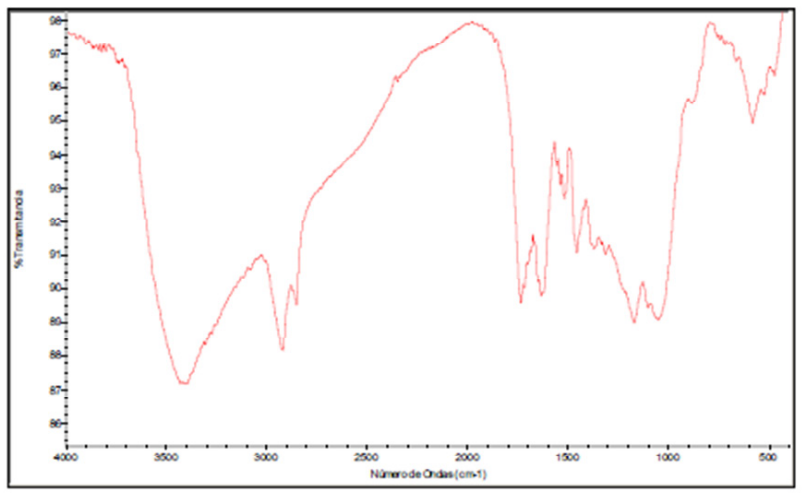

Figura 3: Infrarrojo del bagazo obtenido de la hidrólisis ácida por autoclave (Eucalyptus Camaldulensis).

En el espectro infrarrojo del bagazo tomados en $\mathrm{KBr}$ del E. Camaldulensis resultante del proceso de hidrolizado no presentó diferencias significativas con respecto al análisis realizado al espectro del material vegetal, sin embargo se registra una pérdida o rompimiento de $\mathrm{OH}$ después del tratamiento observado en la primera banda, en este caso la banda presenta una frecuencia de $3400 \mathrm{~cm}^{-1} \mathrm{OH}$ (diferente al grupo $\mathrm{OH}$ del agua), pero no es tan ancha como la presentada en el material antes del proceso de hidrolizado, deduciendo así que el material no sufre cambios estructurales significativos.

\subsubsection{Hidrólisis ácida (ultrasonido):}

La caracterización fisicoquímica efectuada al segundo hidrolizado se realizó con el fin de identificar posibles cambios o similitudes con el material vegetal, el cual fue sometido de igual manera a los mismos análisis fisicoquímicos. En la Tabla 3 se muestra los resultados de esta caracterización.

Tabla 3: Propiedades fisicoquímicas del hidrolizado de E. Camaldulensis obtenido por hidrólisis acida en ultrasonido. 


\begin{tabular}{cc}
\hline HIDRÓLISIS ÁCIAD ULTRASONIDO \\
\hline o Brix & 3.70 \\
$\mathrm{pH}$ & 2.57 \\
$\%$ Acidez & 1.42 \\
Densidad & $1.25 \mathrm{~g} / \mathrm{mL}$ \\
FTIR (Bagazo) & Figura 4 \\
\hline
\end{tabular}

El valor de sólidos solubles es de 3,70 valor cercano al obtenido para el material vegetal. Sin embargo se muestra una disminución de 0,3 , siendo este uno de los parámetros que mas difiere con el análisis realizado al hidrolizado por autoclave. El $\mathrm{pH}$ fue de 2,57 y la acidez de $1.421 \%$ valores referentes al medio en el cual se realizó la hidrólisis. En cuanto al valor de la densidad 1,257 gr/mL se determinó para posteriores cálculos de calor latente.

\subsubsection{Cuantificación de azúcares reductores por DNS}

En la Tabla 4 se muestran los resultados para cada proceso del hidrolizado en autoclave y ultrasonido que son referentes importantes para la posterior fermentación.

Tabla 4: Concentración de azúcares reductores presentes en los hidrolizados

\begin{tabular}{lc}
\hline \multicolumn{1}{c}{ Muestra } & $\begin{array}{c}\text { Concentración Azúcares } \\
\text { Reductores g/L }\end{array}$ \\
\hline Hidrolizado autoclave & 6.709 \\
Hidrolizado ultrasonido & 2.798 \\
\hline
\end{tabular}

El análisis de azúcares reductores muestra que la concentración de los azúcares del hidrolizado por autoclave es mayor que el hidrolizado por ultrasonido, sin embargo el tratamiento por ultrasonido genera un mayor rendimiento en cuanto a la obtención del hidrolizado, y después del proceso de detoxificación la concentración de azúcares reductores tiende a aumentar.

Por otra parte, en la Tabla 5 se muestran los resultados después de la detoxificación realizada al material vegetal.

Tabla 5: Concentración de azúcares reductores presentes Hidrolizado ultrasonido antes y después de la detoxificación

\begin{tabular}{lc}
\hline \multicolumn{1}{c}{ Muestra } & $\begin{array}{c}\text { Concentración Azú- } \\
\text { cares Reductores g/L }\end{array}$ \\
\hline Hidrolizado ultrasonido & 6.798 \\
Hidrolizado ultrasonido & 6.696 \\
Después de la detoxificación & \\
\hline
\end{tabular}

\subsubsection{Fermentación}

Durante el proceso fermentativo se determinó la transferencia de calor a partir de la siguiente expresión:

$$
Q=m C_{P} \Delta T
$$

En donde:

Cp eucalipto: 1, 54 Joule/gr ${ }^{\circ} \mathrm{C}$, (Gilbert, Lazcano, Martin, Vall-Ibsera, \& E, 1997)

$\mathrm{m}$ : masa en gr del material vegetal utilizado

$\Delta \mathrm{T}$ : intervalo de temperatura ${ }^{\circ} \mathrm{C}$

- Secado: Calor (Q) transferido a las 48 horas

$\mathrm{Q}=-2361,74$ Joule

El valor obtenido obedece a la elevada capacidad de absorción que presenta esta especie vegetal, lo cual se ve reflejado con el elevado calor específico que presenta; de tal forma que esto lo hace especial para la producción de edulcorantes y especialmente para el xilitol.

\section{- Hidrólisis:}

Por otra parte, se determinó la energía, en forma de calor que se ha liberado (o perdido) durante la hidrolisis, a partir de la ecuación (1):

$$
\mathrm{Q}=1203,41 \text { Joule }
$$

Comparando los valores obtenido durante el secado y el hidrolizado puede observarse la variación que sufre, siendo menor durante el secado, lo cual esta relacionado con al pérdida de agua ocasionado por el proceso de secado del material vegetal.

\section{- Fermentación:}

Volumen: $70 \mathrm{~mL}$

Densidad: $1,257 \mathrm{gr} / \mathrm{mL}$ Mediante la expresión:

$$
p=\frac{m}{v}
$$

En donde $\rho$ : densidad del hidrolizado

$\mathrm{m}$ : masa del hidrolizado en gr

$\mathrm{v}$ : volumen del hidrolizado en $\mathrm{mL}$

Se tiene que, $\mathbf{m}=87,99$ gr

A partir de la ecuación se obtiene que:

$\mathbf{Q}=1355,04$ Joule

Este valor ganado obedece a la cinética que sufren las levaduras y que esta directamente relacionada con la 
energía de activación del proceso fermentativo.

\subsubsection{Análisis y producción de xilitol}

3.1.6.1. DNS La tabla 6 muestra los resultados de los azúcares reductores durante el proceso de fermentación.

Tabla 6: Variación de azúcares reductores durante el proceso de fermentación Saccharomyces Cerevisiae

Saccharomyces Cerevisiae

\begin{tabular}{lc} 
Hora & Concentración Azúcares Reductores g/L \\
\hline 0 & 6.696 \\
6 & 5.749 \\
12 & 4.730 \\
24 & 4.134 \\
30 & 3.253 \\
\hline
\end{tabular}

En la figura 5 se observa el comportamiento de la xilosa durante el proceso fermentativo, realizado por la levadura Sacharomyces Cerevisiae.

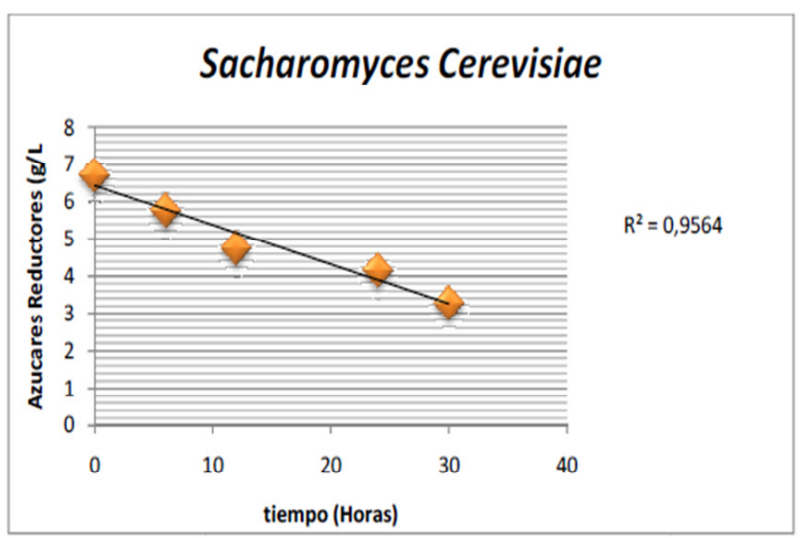

Figura 5: Comportamiento de azúcares reductores durante el proceso de fermentación realizado con Sacharomyces Cerevisiae

A las 0 horas de fermentación el fermentado contó con una concentración de azúcares de 6,6966 gr/L, al transcurrir 6 horas de fermentado dicha concentración empezó a disminuir y se registró un valor de 5,7499 $\mathrm{gr} / \mathrm{L}$ lo cual permitió evidenciar que las levaduras consumieron la xilosa, en los siguientes muestreos correspondientes a las 12, y 24 horas el descenso de la concentración se siguió presentando pero no de manera significativa ya que disminuyo unos $0,595 \mathrm{gr} / \mathrm{L}$, a la hora 30 de fermentación se registró un valor de concentración de 3,2530 gr/L, lo que quiere decir que de las 0 horas a las 30 horas de fermentado la disminución fue de 3,443 gr/L, valor que correspondió al $51,42 \%$ de xilosa consumida por la levadura.
La Tabla 7 muestra los resultados del proceso fermentativo con la levadura Candida Kefyr.

Tabla 7: Variación de azucares reductores durante el proceso de fermentación C. Kefyr

\section{Cándida Kefyr}

\begin{tabular}{llcl} 
Hora & Concentración Azúcares Reductores g/L \\
\hline 0 & \multicolumn{5}{c}{6.696} \\
6 & 5.333 \\
12 & 4.996 \\
24 & 4.291 \\
30 & 4.231 \\
\hline En la figura 6 se observa el comportamien- \\
to de la xilosa durante el proceso fermen- \\
tativo, realizado por la levadura C. Kefyr.
\end{tabular}

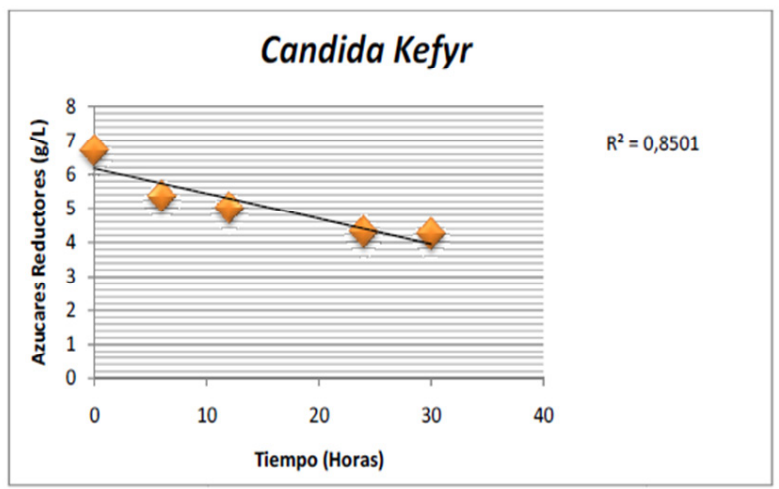

Figura 6: comportamiento de azúcares reductores durante el proceso de fermentación realizado con Candida Kefyr

A las 0 horas de fermentación el fermentado contó con una concentración de azúcares de 6,6966 gr/L, al transcurrir 6 horas de fermentado la concentración empezó a disminuir y registró un valor de 5,3332 gr/L resultado que evidenció que las levaduras consumieron la xilosa, en los períodos comprendidos entre las 12 , y 24 horas. A la hora 30 de fermentación se registró un valor de concentración de 4,2316gr/L, lo que quiere decir que de las 0 horas a las 30 horas de fermentado la disminución fue de 2,465 gr/L, valor que corresponde al $36,81 \%$ de xilosa consumida por la levadura.

\section{CONCLUSIONES}

- A través de los análisis fisicoquímicos realizados a la especie E. camaldulensis se pudo determinar que es una especie de fácil manejo con excelentes propiedades para la obtención de edulcorantes debido a la concentración de azúcares que posee, 
además su capacidad calórica la convierte en un material ideal para la producción de este compuesto.

- El proceso de hidrólisis por ultrasonido presentó adecuadas propiedades fisicoquímicas para la posterior fermentación, debido a que fue un proceso en el cual no se utilizaron temperaturas elevadas que afectaran la estabilidad de los compuestos presentes. Además es el proceso que mejor rendimiento tuvo en cuanto a obtención de hidrolizado.
- El balance de energía realizado mostró que durante el proceso de fermentado hubo ganancia de energía gracias a la acción de las levaduras que metabolizaron la xilosa.

- La levadura que presentó un mejor porcentaje de eficiencia en el consumo de xilosa para la producción de xilitol fue la $S$. Cerevisiae consumiendo en un tiempo de 30 horas un $57,42 \%$, mientras que la $C$. Kéfyr consumió en el mismo tiempo solo un $36,81 \%$ de la xilosa presente en el hidrolizado.

\section{BIBLIOGRAFIA}

1. Boulton, C. A. \& Ratledge, C. 1984. The physiology of hydrocarbonutilizing microorganism. Top. Enz. Ferment. Biotech. 9: 11 - 39.

2. Benjumea R., M. V., \& Correa G., I. (2004). Revistas cientificas- hacia la promoción de la salud- Universidad de Caldas. Retrieved septiembre 29, 2011, from Revistas cientificas- hacia la promoción de la salud- Universidad de Caldas:

3. http://promocionsalud.ucaldas.edu.co/downloads/Revista\%206_6.pdf

4. Buitrago Estrada, J., \& Escobar Romero, A. M. (2009). Aplicación de la levadura Candida spp. Como una alternativa viable para la retardación en la pudrición del banano (mussa acuminata). Trabajo de grado, Pontificia Universidad Javeriana, Bogotá.

5. Escriche RI, S. B. (1996). Toxicología Industrial de Alimentos. Valencia- Universidad Politécnica de Valencia, 242-248.

6. Fernández López, J. A. (2007). Fundamentos del Análisis Cromatográfico. Grupo de Investigación QUIMYTEC UPCT, Departamento de Ingeniería Química.

7. Gilbert, V., Lazcano, R., Martin, F., Vall-Ibsera, \& E. (1997). Trabajos en madera. I.

8. González Hernández, J. C., Álvarez Navarrete, M., Ornelas Hernández, L. d., \& Zamudio Jarami1lo, M. A. (2011). Producción y Aplicaciones Biotecnológicas del Xilitol. Biotecnología, 15(2).

9. Herazo Camaño, I. C., Cárdenas Ruiz, D., \& Arrazola Paternina, G. S. (2011). Utilización de Candida guillermondii aislada del corozo chiquito (Bactris guineesis) en la producción de xilitol. Revista colombiana de biotecnología.

10. http:/www.tropicos.org/name/ consultado en diciembre 12 de 2012

11. Juan Carlos González-Hernández, M. A.-N. (2011). Producción y Aplicaciones Biotecnológicas del Xilitol. Biotecnología.

12. Juanico Loran, J. A. (2004). Síntesis y Caracterización de Nanofibras de Carbono para su aplicación en la adsorción de gases tóxicos. Tesis maestría, Universidad Autónoma Metropolitana, División de Ciencias Básicas e Ingeniería, ciudad de México.

13. Martínez, E., Villarreal, M., Almeida e Silva, J., Solenzal, A., L., C., \& S.L., M. (2002, Diciembre). Uso de diferentes materias primas para la producción biotecnológica de xilitol. Ciencia y Tecnología Alimentaria, III (005), 295-301. Murillo, R., \& Gómez, R. (2006, mayo 08). Espectroscopia infrarrojo. Retrieved abril 15, 2012, from espectroscopia infrarrojo: http://sistemas. fciencias.unam.mx/ fam/Infrarroja.pdf

14. Mussatto, S. I., \& Roberto, I. C. (2002). Xilitol: Edulcorante com efeitos benéficos para a saúde humana. Revista Brasileira de Ciencias Farmaceuticas, 401- 402.

15. Odds, F. (1988). Candida and candidosis. Baillieri Tindall.

16. Orwa, C., Mutua, A., Kindt, R., Jamnadass, R., \& Anthony, S. (2009). World Agroforestry Centre. Retrieved mayo 11, 2012, from World Agroforestry Centre: http://www.worldagroforestry.org/ 
treedb2/AFTPDFS/Eucalyptus_camaldulensis.pdf

17. Placido, M., \& Alemán M, P. (2002). Método higrométrico rápido para determinar la actividad de agua. Ciencia y Tecnología Alimentaria, 230.

18. Santos de la cruz, E., Cancino Vera, N., \& Yenque de Dios, J. e. (2005, enero). El Ultrasonido y su Aplicación. Retrieved Julio 25, 2012, from http:/www.scielo.org.pe/scielo. php?pid=s1810-99932005000100005\&script=sci_arttext

19. Vanegas C., I. A., Yepes P., M. d., \& Ines, D. R. (2004). BUSQUEDA DE CEPAS COLOMBIANAS DE LEVADURAS PRODUCTORAS DE XILITOL. REVISTA DE BIOTECNOLOGIA, II(1), 29.

20. Vanegas Córdoba, I. A., Yépez Pérez, M. d., \& Ruiz Villadiego, O. S. (2004). Producción de xilitol a partir de levaduras nativas Colombianas. REVISTA COLOMBIANA DE BIOTECNOLOGIA.

21. Villalobos Cortinez, V. A. (2010). Comparación de pretratamientos en residuos forestales para la producción de bioetanol de segunda generación: Hidrólisis acida y líquidos iónicos. Tesis Maestría, Universidad de Chile, Departamento de Ingeniería Química y Biotecnológica, Santiago de chile. 